\title{
AKWĒKRÃWAIHKU NÃHÃ ROWASKUZE
}

\author{
XERENTE ${ }^{1}$, Silvino Sirnãwẽ \\ Pesquisador da Ação ‘Saberes Indígenas na Escola
}

\section{ĨSIMÃZUSZE KTURÊ}

Kãhã hâisuka ĩt kakuĩkre tô wakrãiwaihku nã kãtô romwaihku aimõ sissum snã krhêmba mnõ nmẽ, wanĩm akwẽ nõrĩ, kãtô akwẽ wadi aimõ tkrê samãr waihku psê mnõ pibumã. Ahâmre tô Warã, are tâkãnnĩ tô rowahtuze taimõ Warã nõkwakrê wamã krsimãsa. Warã wa tô wawẽ nõrĩ aire kbure rowahdu wanĩm aikte nõrai mã tkrê krtmã rowahtukw, are tãkãnnĩ tô rowahtuzem hawi tet aimõ rowahtukwai nõrĩ kbure wanĩm aikte nõrai mã kbure romkmãdâ tkrê tmã rowahtukw. Aikde tô srõwa hawi za dure rowahdu tê waihku nnãkrat, tapari za rowahtuzem hawi aimõ rowahdu tê kmã sizus mnõ pibumã, tahawi za wnĩm aikte nõrĩ wanĩm romkmãdâ tkrê sawikw, are dure krmã simãzukw wat rmẽ kõ pibumã.

Damrmẽ ĩkuitabi: Warã, wakrãiwaihkuze, romwaihku, wawẽ nõrĩ, rowahtuze, aikde.

\section{RESUMO}

Este artigo tem como objetivo principal apresentar uma reflexão e relatar os saberes do meu povo Akwẽ. Os nossos velhos contam que, desde antigamente, as crianças Akwẽ crescem com sabedorias e conhecimentos que começam de casa e depois vão para o Warã. O Warã era a escola verdadeira do povo Akwẽ, onde ficavam os professores que eram os anciãos. Atualmente o Warã foi substituído pela escola que a maioria das aldeias tem. São ensinados todos os saberes às crianças, sobre sua cultura, e, também, a valorizarem seus costumes, e a saberem conviver no meio do seu povo, com os não indígenas. Devido à escola, eles se tornarão os melhores prefesssores, para depois passarem todos os saberes do seu povo e, então, formar outros jovens.

Palavras-chave: Warã. Saberes. Anciãos. Escolas. Crianças Akwẽ.

1 Rede UFG/UFT/UFMA. Professor de Educação Básica. 


\section{ABSTRACT}

This article has as main purpose to present a reflection and to report the knowledge of my Akwẽ people. Our elders tell us that Akwẽ children have grown up with wisdom and knowledge since the old days, starting at home and then going to Warã. The Warã was the true school of the Akwẽ people, where the teachers who were the elders were today replaced by the school most villages have. They teach all the knowledge to the children, about their culture, and also to value their customs, and to know how to live among their people, with non-Indians. Because of the school, they will become the best teachers, so they will pass all the knowledge of their people and then train other young people.

Key words: Warã. Knowledge. Elders. Schools. Akwẽ Children.

Ĩptokrta nõrĩ tô kãnẽ taimõ romkmãdâahâmre hã tkrê waskukw, akwẽ nõrĩ tô krãiwahku snãtet aimõ krkuiput, tô aiktere hawi za aimõ krãiwahkuk. Ahâmre akwẽsĩm mãrd Warã krtabnã dat krwamtrẽ mnõ, tô Warãhawi taimõ krwatbremĩ nõrĩ, kãtô baktõ nõrĩ tetaimõ krãiwaihku snã kuipktuk. Ahâmre Warã tô rowahtuzem krtabi akwẽ nõrai mã, tazi za wawẽ nõrĩ kahâ snã krhêmba tô siwawi mnõ pâ za aikte nõrai mã romkmãdâ tkrê waskukw, aretahã kwatbremĩ nõrĩ tô siwawi mnõ pâ awãrê sĩm mãr mnõd, tô sisdanãrkwai mnõ pâ za ku aimõ tê awãrê tê sõrekw.

Tâkãinnĩ tô rowahtuze nha dazakrui mbamãrd tanẽkõwa tetôaimõ krhêmba, kãtô dure rowahtukwai nã dat krwamtrẽ mnõ, tanõrĩ tô wawẽnõkwakrê taimõ wanĩm aikte nõrai mã tkrê tmã rowahtukw, ahâmre tô Warã ktabi, are tâkãnnĩ tô rowahtuze tet aimõ krwam simãsa. Wawẽ nõrĩ nãt aimõ rowahdu aikte nõrai mã tkrê tmã waskukw, tahã tô Warã nã dat krwamtrẽ mnõ. Tahã Warã hawait taimõ krwatbremĩ nõrĩ kãtô baktõ nõrĩ ro kahâ snã tkrê waihkukw, kãtô tkrê samãr waihkukw. Ahâmre tô Warã hawit tet aimõ romkmãdâwanĩm aikte nõrĩ kbure tkrê kwaihkuk, kãtô dure danrõwa hawi za dure aikte ptokwa, kãtô ĩseparkwa kbure romkmãdâ kra nõrai mã tkrê tmã waskukw.

Aikterê tô separkwai dkikrê wamre za ro kbure tkrê wapar, mãrĩ ĩseparkwa tê kmãkwamãr waza dure kra mã rowaihu tmã wasku, tanẽkõwa mãrĩ ĩserpa tê kmẽsi wa za dure kra mã tmã rowahut, ĩseparkwa damẽ sõkrê wa za dure aikterê danõkrê damẽ wapa, ĩseparkwa sai psê snã krsai mnõwa za aikteprerê hâiptorãrê snã twaptkãrê, are ĩseparkwa sai prãirê snã krsai mnõwa za dure aikteprerê hêmba prãirê snã twaptkãrê. 
Ĩseparkwa mãr kunẽ tkrê kmẽsi mnõwa za aikteprerê mã psê kõdi, mãrĩ ĩseparkwa tê kmãdkâ wa aikterê dkiwa tkrê dur re kbure rotkrê kmãdkâ mnõ za aikteprerê waptkãrê wi nẽs siza, tahã tô wanẽku, amke, kãtô rom wadi mnõ. Tanẽnmẽ tokto waptkãrê pari tô spokrê nã za aimõ tkrê dawapar, kãtô tmõ nã za rokbure tkrê krmãdârê, aikterê tô kuiptu mnõ pra, za ro kahâ tê kawahkurê, tanẽnmẽ tokto kuipturê wi za sĩsrĩ kmãsnãkrat, tahã tô separkwai mã, ptokwai mã kãtô dure dawadi nõrai mã, tapari za siwaprossi za krsimãssirê, tapari mãtô tokto ro sismĩstê tkrê kadur, aimõ za tkuiput, aimõ za dure îkrãiwaihkuze dawer, tanẽsnã za aimõ îkrãiwaihkuze tmã tkuiput.

Aikterê za waptãr, pikõirê tanẽkõwa ambârê, za tkuiput are ro tkrê samãr waihukw, aikde tô ro kmã sapka prabâ zaro tkrê kmãkwamãr, ĩseparkwa tanẽkõwa ĩptokwa ro tkrê tmã wasku mnõ kõwa za aikte nõrĩ sipkẽ nã kwrahkukw. Tanẽnmẽ arê wanĩm aikte mã ro wat krwasku sku kba mõnõ, aimõ krãwaihku snã krwrahku mnõ pibumã. Aikte mã tô ro wat krtmã wasku mnõ hawi, za aimõ aikte nõrĩ tkrê dazazê, are dure aisa dat krsazê, aikde tô smĩwapar hawi za ro tkrê samãr wihku pês, kãtô smĩzazêzem hawi za dure kbure tkrê dawaze, ĩptokrda kãtô aikte sikuwa wapte za tkrê zazê, are dure aisa dat krsazê.

Akwẽ kra wi za pkẽtoi wawẽ, mrõ mẽ, kãtô ĩsim akwẽ nõrĩ, ambâ hawim hã, kãtô pikõi hawim hã, tanẽnmẽ za sissum snã za sihdurê sapar psêkw, tô sissum snã za sihdurê tkrê sapar psêkw, tare za tkrê waikhâkw aikterê sissum snã psê snã aimõtê wrahku mnõ pibumã. Akwẽ kra tô srurêre hawit taimõ rotkrê wapar, kãtô tmõ nã za aimõ ro tkrê krmãdâk. Warã tô dure dakrãikwaihkuze, tô Warã hawi kãtô dure srõwa hawi za aikte nõrĩ kbure ro tê waihuk tô Warã hawi kãtô srõwa hawit, taimõ aikte nõrĩdasĩpê dasiwawi, danĩpi, danõkrê, dasiwaze, romkrêptkã, dasiwawmtrẽze, sasari, tpêkwapsi kãtô romwadi mnõ kbure tkrê samãr waihkuk.

Aiktepre waptkã wi za kbure sapar psên, akwẽ nĩm rokmãdkâ wa, akwẽ kra snĩ ambârê dam sapar psê krtabr, tô ahâmre hawi tet aimõ ambârê dam sawi krtabdi. Ambâ nõrĩ nha kra snĩ ambâ wa tô dam kmãdkâ psê snãza dat krmãdâk, dam sazêi snã za aimõ dawadi nõrĩ tkrê kmãdâk. Tôtahã tô aimõ kra snĩ ambâ kahâ nmẽ, tô aimõ ahâmre hawi aimõ wahĩkba nõrĩ krsikwapeĩ kba mnõ nmẽ. Ahâmre nãt aimõ krsissô krwaireb krsikwapeĩ kba mnõ pibumã, kãtô dure danĩpi hãwa azanã snã tkrê kmã wazrê kba mnõ pibumã, kri zabu hãwa za dure azanã snã sabukw. 
Tanẽ kõd pikõirê nã pikõ kra wa, za kmãdkâ wapu snã za baktõrê dat krmãdâk ahâmre wahikba nõrĩ tkrê wasku kba mnõze, pikõi nõrĩ tô sakukrêsi, are tâkãinnĩ ĩkwaimba kõdi, wanõr mã, pikõi nõrĩ dure sĩm romkmãdkâ psêdi, tanõrĩ za dure sĩm ambâ tkrê waihâk, bru mba, kri wa, sasar mba za mrõ mẽ krmõ, tpêkwapsi mba za dure krmõ, kãtô kra mã za dure danmĩzazêzem nã tmã rowahut, aimõ smĩzazêi snã wrahku pibumã.

Tahã tô wanĩm pikõi nõrĩ aimõ knmrmẽ mnõ kõhawi kãte, tô ambâ nõrai wtẽsi mrmẽprêzem hawi kãte, ambâ nõrĩ za sĩm aikte mã mrmẽprê zawre snã romkmãdâ tkrê tmã wasku, kãtô danĩpi tmẽ za aimõ kra tkrê kwanĩ pês. Aikte nõrĩ tô kbure tô ambâ nõrai tê, mãr wa tô dasiwawi pra, ambâ kra mnõ tô kbure za ptokwai tmẽ krsiwawik, pikõ tô sisdanãrkwa nã za aimõ tmrõ, tanẽnmẽ tô tare za aimõ damã kra, are pikõ dure kra tmã sawidi, tanõĩ tô dure aikte mã rowaskukwa.

Nmãzi za aikterê sipkra nã mãrĩ kupi, tanẽkõwa kmãnã, tô ro tkrê kmãdkâ mnõzem hawi. Aikde kuiptu pari za dawawi waihuk, tô tkrê dakmãdkâ mnõ nmẽ, za srurêre hawi za dawawi waihuk, tazi za tokto sawre pari waptem sikuwa tkrê wawi, sisdanãrkwai nẽhã za tkrê wawi, tanẽkõwa ambâ nẽhã dasĩpsêmba za sisdanãrkwa tkrê têkrã. Pikõ hãwa tô pikõi sikuwa za tkrê wawi, tanẽkõwa tô ambâ zemã za nmãhã nõrĩ wawi.

Tanẽ dure danõkrê, aikde tô tkrê dawapar mnõ hawi za dure nõkrê waihukw, akâ za spokrê nãsi tkrê dawapar, are nmãzi za tokto kmã tissanõ sõkrpsê zô. Akwẽstôsõkrpsê snã waza tokto damẽ krsõkrê, baktõ nĩsi nã, kãtô kwatbremĩ nĩsi nã, zâ nã kãtô danõkrê wadi nã za ambâ hãwa damẽ krsõkrê. Ambâ nmãhã sõkrpsê waza aimõ smĩstun danõkrê tkrê dam kwanĩ mnõ pibumã, baktõ nĩsi nã, kwatbremĩ nĩsize nã, aimõ krdam sõkrê mnõ pibumã. Are pikõ hãwa tô tare za tkrê dawaihâk, pikõi sikuwa za tkrê wawi, tanẽkõwa tkrê dawaihâk.

Dasiwaze tô aiktere hawi za dure aimõ kmã snãkrat, tô rowahtu hawi za dure aimõ tê waihuk, aikte nõrĩ tô waihku snã za tkuiput, tô dasiwawi mnõ pâ taimõ wanĩm aikte nõrĩ krsawer. Ĩtdêkwai mnõ pâ za aimõ kra mã dasiwazezem nãhã ro tkrê tmã waskuk sipranẽ wasiwawize ĩhêmba, are wanĩm aikde tô srurêre hawi za aimõ romkmãdâ waihku snã re dawer. Aikterê tô waihku snã za sisdanãrkwa aimõ krmãsõpre, nõkwa ĩsisdanãrkwai nã. Tahã tô ĩptokwai hawit, kãtô ĩseparkwai hawit taimõ dasiwaze tkrê kmãdkâ psêkw, kãtô dure romkrãikrda kra mã tkrê wasku. 
Dasiwawize tô krdasiwazem mnõ pibumã, dasiwawi hawit taimõ dure dasiwamtrẽzem nã aimõ krdassanãmr, kãtô dure dassazêzem pibumã taimõ krdasiwawi.

İzeparkwa tô ĩnatkû nã waza aimõ ît krwamtẽr, are ĩptokwa tô ĩmmã nã waza aimõ ît krwamtẽr, ahâmre tô tâkãnẽ waza aimõ wat krsanãmr, tanẽkõd tokto tâkãinnĩ tô ktâwamkõ mrmẽzem nã wat aimõ wazeparkwa kãtô waptokwa wat krwamtẽr. Tôtanẽ dure ĩmmã, tahã tô ĩptokwai zdekwa, tanẽkõwa ĩptokwai pnã, waza aimõ ĩmmã nã ĩt krwamtẽr, are aisa tô bremĩ za tkrĩ wamtẽr, are pikõ hãwa tô baknõ nã za saihâ. Are krawaptem kra hãwa tô ambâdi, nã tanẽkõwa pkõiti nã za saihâ, ambârê hãwa tô ambâdirê za tkrê wamtẽr, are pikõirê hãwa tô pkõitirênã za tkrê wamtẽr.

Danĩmnõkrêzu tô pikõ ambâ hidba hawi taimõ krhêmba, ta nẽnmẽ za aimõ romkmãdâ simã tkrê kmãdkâ psêkw. Ambâ pikõi nã krawa za aimõ wahidba hawim hã ambâ ĩdumkrta mã za kratmã sakre simã ktrê kmãdkâ psê mnõ pibumã. Wahidba pikõi rênã kra wa za dahitbre wdênrõ sda kawazu are kmã sõkrwassis, simnõkrêzuinẽ za tkrê kmãdâk, tahã wdênrõ tô amrẽsku, nmãzi mrõ wa tô simnõkrêzu zda bâ za dasa zô krmõ, aikuwa za kbazêiprãi zô za krmõ, za nẽs sô dako mãranã, tanẽkõwa tpê zô za krmõ, simnõkrêzui mã kbahi tê kmãkrãinistu mnõ pibumã, (dakukâ), ahâmre dakukâ tô kupakbu zbrê nã, tpê prarõr nã za simnõkrêzui mã romkmãdâ tê kmãkrãinĩsut. Danĩmnõkrêzu snĩ ambâ tanẽkõwa danĩmnõkrêzu sĩm pikõkrawa, ambârêhãwa za ambâdi nã za tkrê wamtẽr, are pikõi nã kra wa za dure pkõiti nã za tkrê wamtẽr, tô aimõ krdasiwazem mnõ hawi. Are aisaza dure aimõ danĩmnõkrêzu, nõkrêkwa nã tkrê wamtẽr, pikõ kãtô ambâ za aimõ tanẽsnã tkrê wamtẽr, kãtô îkrda nã za dure aimõ tkrê wamtẽr. Tanẽ dure danĩhdu nõrĩ za ptokwai nĩmnõkrêzukwa za îkrda nã za tkrê wamtẽr, kãtô tahã ĩsimpikõ za dure îkrda nã za tkrê wamtẽr.

Aikteprerê za dure srurêre hawi za dure dat krwawi: ambârê hãwa za pat nẽ za wawin, are pikõirê hãwa tô tare za dat kumtorê, huku nẽ. Are baknõ kuiptu wi za dure ktâ nẽ za dat krwawi, aimõ aikte sikuwa waihku snã krmã sõpre mnõ pibumã. Tahã aikde dat kumto mnõ tô dasĩpsê mba si za dat krwawi, tahã za dure aikte sikuwa arkiwi krmã sõprekw.

Akwẽstô tanẽnã aikde dat wawi kõwa tô ĩsiwawizem nã za wawin, kuzâp kra hãwa za kuzâp siwawizep nã za wawin, wahirê kra hãwa za wahirê siwawizep nã za wawin, kbazi kra hãwa tô kbazi siwawizep nã za wawin, ĩsake kra hãwa tô ĩsake siwawizep 
nã za wawin, are krito kra hãwa tô krito siwawizep nã za wawin, are krãiprehi kra hãwatô krãprehi tô ĩsiwawizep nã za wawin. Aikde dure wamtrẽzepti, tô wamtrẽ mnõ pâ za aimõ dat krwamtẽr. Ambârê hãwa tô turêrê nã za dat krwamtẽr, are baktõrê hãwa za tarêrê nã za dat krwamtẽr, are tanõrĩ kuiptu pari za ĩwamtrẽze dure aimõwi snã za dure saihrân

Kãhã za kbrure dakrãiwaihkuzem wa kawakõi pês, tô aimõ krmã wasiwrahku mnõ pibumã nãt aimõ ahâmre hawi ĩptokrta nõrĩ romkmãdâ tkrê sakram psêk, aimõ tô tanõrai pradikuirĩ nã krmã wanpokrku kba mnõ pibumã. Aikterê wat krsawi kba mõnõ, tanõrĩ tô kahâ snã za wahu adu kmã tinẽ, are tanõrĩ za dure kra nõrai mã kãtô sihdu nõrai mã romkmãdâ tkrê wasku psêkw.

Romkrêptkã dure mãrdi, tahã kbure dam waihku kõdi, tô nõkwa mnõrai wtẽsi za waihuk, ambâ kãtô pikõ za romkrêptãk, are tô nõkwamnõrai wtẽsi za krsikwasi. Romkrêptkã krêmba mnõ tô dasisnã damrõ wamsi, rowahdu wawẽ tê sõmr wa, dakrãi wakbâze, dasikrãiktõ mba, tanẽkõwa dasipsê mba za aimõ wawẽ nõrĩ sisdanãrkwai mnõ pâ simã romkrêptãk. Romkrêptkã wẽ snã za nõkwa wapa, are kbure dure dam samãr waihku kõd, wawẽ nõrĩ tô wawẽ mrmẽzem nã za aimõ romkrêptãk tazi aikterê nõrĩ, waptem nõrĩ kbure tê samãr waihku kõdi. Wawẽ hawit taimõ romkrêptkã dat krwaihuk, kãtô dure danmĩwapar hawi za aimõ nõkwa romkrêptkã waihuk.

Sasari dure akwẽ nõrĩ tmã wẽki akwẽ kra tô sasar mba za dure dawer, tô ahâmre hawit taimõ akwẽ nõrĩ sasar mba krwareb, tanẽnmẽ aikterê nõrĩ tô srurêre hawit taimõ sasar mba dure damẽ krwareb, nmãhã dazerparkwai nõrĩ wasã snã za damẽ krmõ, tanẽkõwa waikrê snã. Ahâmre tô dakahâ snã taimõ sasar mbakrnõmr, nha ptâ ssõre nã za aikuwa mba krnõmr, awãrê za aimõ tê sõrekw mãranã nẽs nwa stõ kba mnõ pibumã. Ambâ nõrĩ za nẽs awẽre tê sasar kbazêiprã tkrê smrõ mnõ pibumã, are pikõi nõrĩ za nẽs ssu tê wazu, skrã, siknõ, waptâ za dure pikõi nõrĩ tê kmãkwamãr, aipâ srõwa ku wahtu wa, ĩnĩ zaza tê nwa kwasar pibumã. Siktõ wa za dure nõkwa mmĩ nwa kadu, kupa, knẽ, kãtô aikterê za dure nõkwa nwa du.

Tpêkwapsi mba za dure aikterê ro kbrure tê waihuk nhanẽ tpêkwapsi dat kmãnãr mnõ, aikte nõrĩ tô srurêre hawi za aimõ tpêkwapsi mba krnõmr. Are tpêkwapsi mba siwakrum kõdi, pikõ wasã temrêre tpêkwapsi mba damẽ krmõr kõdi, tanẽ dure ambâ hãwa dure damẽ krmõr kõdi, tanõrĩ tanẽmba dam sipaki, mãr wa tô wasãzem (waikrê wa) hawi za aimõ kunã psên. Ambâ hãwa za aimõ kunã psên aimõ wawẽ nõrai smĩ rowahdu tê wapar pibumã. 
Tô dazakum sisi za aimõ krmõ, tahã damẽ krmõr wa za kbawazi tê daw siptê prãirê, tanẽkõwa kbawazi kmã siptê prãirêki, kbawazi siptê prãirê wa za tbê tê smrõi mnõ kõdi, tare za ku tbê tê topre wawẽ, tazi za tbê dat smrõ mnõ kõdi, tpêkwapsi wẽki tainnĩ za aimõ ambâ nõrĩ tbê wakrowdê nã siw tê hâzu, kãtô smĩkezem nã za aimõ dat smrõ, kãtô pikõi nõrĩ za dure smĩkezem nã za aimõ tbê tê smrõi wawẽ, kãtô wapte, aikte nõrĩ za dure tbê tê smrõ.

Aimõwi za pari wa tbê trêrkê wawẽ, tahã kbawaski wanã za aimõ ssakrê, tanẽnmẽ tô psê snã za aimõ trêrkê wawẽ, tahã za kbure kmã tasĩmprumn, wapte mnõrĩ, kãtô mrõre nõrĩ, kãtô ĩssuibka nõrĩ, za aimõ kbure kmã kawakõin. Are tahã pari zdawawa za dure akwẽ snĩ ambâ, tanẽkõwa pikõi nẽhã za aimõ nãmr tpêzô smĩpar snã, are tô romwaihkukwai nẽhã, tanẽkõwa tô akwẽ ĩpkẽ psê nẽhã za aimõ nãmr are awẽre hã za dure kmãdâk. Tpêkwapsi tô kbawazi nã tet aimõ dat krwapsis, tpêpãrze, kãtô wdê aikuwa mba hãnã za aimõ tbê kmã kakwapsin.

Tokto tô wanmĩ rowahtuzem nã waza kakuĩkre, tâkãnnĩ tô rowahtuzem hawiwat aimõ romkmãdâ kahâ snã wat kawaihuk, kãtô dure wanrõwa hawi waza dure wakra mã romkmãdkâ pê kbure wat krtmã wasku, are dure romkmãdâ îkunẽ nẽhã wat ktwi wẽkõ. Dakrãiwaihkuze, kãtô romwaihkuzetetô aimõ sissum snã krhêmba, tanõrĩ za kbure romkmãdâ wamã wasku pês, tâkãnnĩ rowahtuze tetô aimõ dazakrumbakahâ snã krhêmba, nharê dazakru tetô rowahtuzem krnãthêmba, are tetô aimõ dakmãdkâkwai nõrĩ, kãtô wawẽ nõrĩksrô krsikwanĩkw aimõ dazakrui mnõ pâ rowahtuze simãsam mnõ pibumã.

Aikde za kbure rompê, kãtô romkmãdkâ pê rowahtuzem hawi tê waihuk, tô tahawi za dure danĩpiĩ pibumã krsikwanĩk, nmãzi danĩpi nmãhã hâisuka zanãmrkwai nõrĩ tê kmẽsãmr kba wa, aimõ psê snã krsipiĩ mnõ pibumã, kãtô dure wanĩm romkmãdâ tkrê sawi kba mnõ pibumã za rowahtuzem hawi kbure rowahtukwai nõrĩ romkmãdkâ pê wanĩm aikte nõrai mã tkrê waskukw, tanõrĩ tô aikde, tanẽnmẽ wanõrĩ rowahtukwai nõrĩ waza aimõ rowahtuze mba kbure wanĩm aikte nõrai mã romkmãdkâ psê nẽhã wat krtmã wasku. Wanmĩzazêze, wanmĩkuĩkreze, wanmĩromkmãkwamãrĩ, wamrmẽze wasiwawize, wasiwazemze, wasiwamtrẽze, danõkrê, sasari, tpê nõ, danĩpi, wamnõwa, dazakru, kãtô romwadi mnõ, aimõ wat krsawi mnõ pibumã, kãtô dure kbure wanĩm aikte nõrĩ tkrê sawi mnõpibumã za aimõrowahtuzem mba rowahtukwai nõrĩ kbure wanĩm aikte nõrai tkrêwaskukwa, aimõ krmã spokrku kba mnõ pibumã, are dure aikte 
skuwa mã, kãtô dawadi nõrai mã tkrê wasku kba mnõ pibumã, tahã rowahtuze tô dakrãiwihkuze.

Tâkãnnĩ tokto tô rowahtukwai nõrĩ za aimõ wanĩm wawẽ nõrai sõkwakrê ksimãsa, mãr wa tô wanĩm wawẽ nõrĩ aimõ prãirê zem hawi, wanĩm wawẽ nõrĩ mãtô nharê aimõ tsirmẽk, tanẽnmẽ arê wanõrĩ rowahtukwai nõrĩ wanĩm wawẽ nõrĩ wat krsdanãr kba mõnõ, tanõrĩ tô romwaihku tdêkwai ktabi, tanõrĩ tetô hâisuka krãikrta re aimõ dazakrui mnõ pâ krsikburõ, ambâ kãtô pikõi nõrĩ, tet aimõ wanĩm romkmãdâ krãi kba wa kbure tkrê kburõikw, kãtô sakrui mnõ pâ teto kre sikburõ, nmãzi dasĩpsê waza tanõrĩ dasipsê ku krãiwaihkure wahud aimõ wawẽ wadi nõrai mẽ sĩm romkmãdkâ nã aimõ kmã sitom mnõ pibumã.

Wanĩm wawẽ nõrĩ za dasĩpsê mba aimõ kbure romkmãdkâ pê wanĩm waptem nõrai mã tkrê waskukw. Dasĩpsê mba za dure wanĩm aikte nõrĩ, pikõ kãtô ambâ nõrĩ za kuiptu snã romkmãdâ tê kawaihkukw, tahã dasĩpê tô dure rowahtuzem nẽ za ĩptokrta nõrĩ tkrê kmãto, tainnĩ aikte si romkmãdâ tkrê waihku mnõ kõdi, tô waptem nõrai zemã kãtô dure ĩptokrta nõrai zemã, za aimõ wanĩm romkmãdâ tê waihkukw, dasipsê mba za kbure romkmãdâ wawẽ nõrĩ tkrê waskuw.

Tanenmẽ wanõrĩ rowahtukwainõrĩ waza wapkẽto nãkbure wawẽ nõrai mã wasiwaskun, wawẽ nõrĩ tô siwawi mnõ pâ waza aimõ wat krmãdkâ pês, kãtô dure wat krsazê, tanẽzem hawi waza wnĩm wawẽ nõrĩ kuiptu snã wat krsawi, ambâ kãtô pikõi nõrĩ. Tâkãhã romkmãdkâ te dakrãiwaihkuzem nãhã, kãtô dure rowaihku nã nãhã aimõ wat kakuĩkre mnõ, za aimõ rowahtuzem mba za aimõ krsmĩkwar, kãtô dure rowahtuze krikahâzawre mba hã, tô aimõ bdâdi tê wam sdâhui mnõ nmẽ.

Tanẽnmẽ tâkãhã romkmãdâ psê krtabdi, aimõ mnĩ wasiwaikẽ rowahtukwai nõrĩ tê wawaihkâ wa, dure wapkẽto zawredi, tâkãhã romkmãdâ za tare aimõ waw wdakur pês aimõ wanĩm romkmãdâ ptâ bâ wat krsawi mnõ pibumã, kãtô wanĩm romkmãdâ wat rmẽkõ pibumã, tanẽnmẽ watô sapar psên tâkãhã hâisuka krãikrtarê wat kakuĩkre wa. Tô ahâmre hawi tet aimõ dakrãiwahkuze kãtô rowaihkuze sissum snã krhêmba mnõ nmẽ, are wanõrĩ rowahtukwai nõrĩ wat aimõ tokto si wat samãr waihku pês, tô wanmĩ kuĩkre waihku mnõ nmẽ aire, kãtô dure hâisuka tmẽ aimõ krwasikwanĩ mnõ hawi, akwẽ kra, wapte mnõrĩ, kãtô ĩptokrta nõrĩ tô ro wat kmãdkâ mnõ hawi, kãtô dure wanmĩpar hawi waza ro aimõ wat krsamãr waihuk, tanẽnmẽ waza tokto kbure wanĩm romkmãdâ hâisuka krãikrtarê mba kuĩkre snã wat 
sakra mnõ pibumã aimõ krwasikwanĩ, aimõ kuĩkre snã smĩkra mnõ pibumã. Nmãzi dawadi nõrĩ tâkãhã hâisuka krãikrtarê tê sanãmr wa wẽ snã tê sanãmr pibumã, tanẽzem hawi wazatô wanõrĩ akwẽ aimõ wanĩm romkmãdâ wat krsawi pês, are dure wanĩm aikte nõrai ptâ bâ wat krwasku, tanõrĩ dure wabrapâ tkrê sawi kba mnõ pibumã. Twa kãnẽ tenharêtê!

\section{Saberes Indígenas Akwē}

Os nossos velhos contam que, desde antigamente, os meninos e as meninas Akwẽ nascem com sabedoria, vendo as coisas que acontecem no meio do seu povo. Assim, eles vão crescendo e conhecendo a sua cultura. Antigamente, havia a casa dos solteiros, que os mais velhos chamavam de Warã. Na casa, ficavam os professores que conheciam tudo sobre a sua cultura. Esses professores são os anciãos.

No Warã, ficavam os anciãos de todos os clãs. Lá, eles ensinavam para os meninos e as meninas os saberes do seu povo Akwẽ. No Warã, entravam os meninos de 8 a 10 anos de idade, bem como as meninas. A casa era a escola verdadeira, que passava todas as leis e os conhecimentos para ajudar os alunos a se tornarem grandes líderes no meio do seu povo Akwẽ. No Warã, os alunos aprendiam todos os ciclos das vidas das crianças e saíam preparados para conviver com a sociedade no mundo, porque a sabedoria e o conhecimento não se separam, os dois ficam juntos. Ali, eles aprendiam o respeito, a valorização da sua cultura, para que um dia se tornem bons professores e ensinem outros jovens a valorizarem sua cultura.

A metodologia Akwẽ está no olhar, no ouvir, para depois ser colocada em prática. Hoje, nós temos as escolas indígenas no lugar do Warã. Nelas, as crianças Akwẽ aprendem com seus próprios professores Akwẽ, que ensinam na sua própria língua, mostrando desenhos etc. Eles ensinam a escrever na sua própria língua materna e em português, que é a segunda língua. Os alunos aprendem, também, a conviver com a sociedade.

As escolas têm que se colocar no lugar dos pais dos alunos, para ajudar os alunos a aprenderem a sua cultura, saberem valorizá-la e estarem preparados para o mercado de trabalho. As escolas Akwẽ precisam ensinar mais, para os alunos crescerem com sabedoria e 
conhecerem mais sobre o que é a escola Akwẽ - inclusive as diferenças que a escola tem - por meio da cultura, da língua materna, pintura corporal, respeito, festa indígena etc. Todos esses conhecimentos a escola tem que passar para os alunos Akwẽ, para que eles tenham um ensino de qualidade. 